\title{
Frequently cutting restriction enzymes: Clearing the fog to see the ends
}

\author{
María I. Vaquero-Sedas, Miguel A. Vega-Palas \\ Institute of Vegetal Biochemistry and Photosynthesis, CSIC-University of Seville, Seville, Spain \\ Email: vega-palas@ibvf.csic.es
}

Received 30 November 2012; revised 27 December 2012; accepted 4 January 2013

\begin{abstract}
Telomeres, which are found at the ends of eukaryotic chromosomes, are composed of tandem arrays of repetitive sequences and safeguard genomic stability. Previous studies have revealed that telomeric repeats are also present at internal chromosomal loci in many eukaryotes. However, the biological role of these interstitial telomeric sequences (ITSs) remains unknown. The integrity of telomeric length and chromatin structure is required for telomere stability. However, the study of these telomeric features can be impeded by the presence of ITSs. Frequently cutting restriction enzymes have been revealed to be very useful tools for the study of the length and chromatin structure of telomeres independent of the presence of ITSs.
\end{abstract}

Keywords: Telomeric Length; Telomeric Chromatin Structure; Frequently Cutting Restriction Enzymes; Arabidopsis thaliana

\section{INTRODUCTION}

Telomeres usually contain double-stranded tandem repeats of a 6 - 8 bp G/C-rich motif followed by a singlestranded overhang of the G-rich strand. The telomere length is characteristic for each organism and must be properly regulated to ensure telomeric stability [1-3]. This has been clearly demonstrated in studies of mutants lacking telomerase, the enzyme that replicates telomeres [4-6]. In addition, the maintenance of telomeric chromatin structure is also required for telomere stability, even if the telomeric length is not altered $[7,8]$.

The homeostasis of telomere length and chromatin structure is essential to prevent chromosome fusions and degradation by exonucleases as well as to ensure accurate DNA repair, homologous recombination, chromosome pairing and segregation. In mammals, the loss of telomere stability can affect many significant biological phenomena, such as aging, illness and cancer [1-3]. Therefore, the study of telomere length and chromatin structure is of major interest. Ideally, these studies would not be influenced by the existence of ITSs.

The first approach described to study telomeric length in different organisms was the analysis of terminal restriction fragments (TRFs) [9]. In TRFs analyses, genomic DNA is digested with frequently cutting restriction enzymes, resolved on agarose gels and hybridized with a telomeric probe. This approach allows for the determination of telomeric length using appropriate markers. Telomeres are composed of perfect repeats that are not typically cut by restriction enzymes. In contrast, ITSs usually are digested by restriction enzymes. Therefore, ITSs do not generally interfere with the determination of telomeric length [10]. However, TRFs analyses cannot be applied to some organisms, such as the Chinese hamster, in which ITSs are nearly perfect and more abundant than telomeres [11].

Some alternative methods to TRFs analyses that do not involve the use of restriction enzymes have been described to study telomeric length in different organisms [9]: 1) In fluorescence in situ hybridization (FISH), telomeres are labeled with fluorescent probes. This allows for the measurement of the average telomeric length ( $\mathrm{F}$ FISH) in a single cell or the length of each individual telomere in the cell separately (Q-FISH); 2) The analysis of the lengths of unique telomeres (STELA in humans or PETRA in plants) involves the use of telomeric and subtelomeric primers, which permits the study of the telomeric length of specific telomeres; 3) Quantitative PCR (qPCR) involves the use of degenerate telomeric primers and may require the amplification of other genomic sequences as a DNA quantity control. Nevertheless, all of these alternative methods require validation prior to use and are usually validated by TRFs analyses when possible.

The chromatin structure of telomeres has been analyzed with two techniques: immunocytolocalization and chromatin immunoprecipitation (ChIP) followed by dotblotting and hybridization with a telomeric probe. Both techniques generate uncertain results because immunocytolocalization detects both telomeres and subtelomeric regions, while ChIP detects both telomeres and ITSs. 
Even if the same biological system is studied using both techniques, the conclusions obtained might be ambiguous because of the detection of subtelomeric ITSs [12].

Two additional approaches have been described to study telomeric chromatin independently of ITSs. The first technique uses a frequently cutting restriction enzyme and has been used to analyze the chromatin structure of telomeres in Arabidopsis thaliana [13]. Arabidopsis telomeres are composed of repeat arrays of the plant-type sequence (TTTAGGG/CCCTAAA). These repeats are also very abundant at interstitial chromosomal loci [14-19]. The restriction enzyme Tru9I, which recognizes the sequence TTAA/AATT, was used to differentiate between the telomeres and the ITSs [13]. Telomeric repeat arrays at Arabidopsis telomeres are perfect, so they remain uncut after digestion with Tru9I. In contrast, the telomeric repeat arrays of ITSs in Arabidopsis are composed of degenerate telomeric repeats interspersed with short arrays of perfect telomeric repeats $[10,17,18$, 20]. Therefore, Arabidopsis ITSs are frequently cut by Tru9I. After digesting Arabidopsis genomic DNA with Tru9I and hybridizing with a telomeric probe, the hybridization signals corresponding to ITSs mostly disappear, with only three ITSs bands smaller than 500 bp remaining. Therefore, the signals detected above $500 \mathrm{bp}$ after Tru9I digestion correspond only to telomeres. In turn, when Arabidopsis genomic DNA is undigested, the signals detected above $500 \mathrm{bp}$ correspond both to telomeres and to ITSs [10]. This observation allowed for the study of the chromatin structures of Arabidopsis telomeres and ITSs independently. After performing ChIP, the input and immunoprecipitated DNA samples were amplified with a whole genome amplification protocol. Then, the DNA samples, undigested or digested with Tru9I, were resolved in an agarose gel and hybridized with a telomeric probe. Finally, the relative enrichment of ITSs versus telomeres was calculated [13].

Using this method, Arabidopsis telomeres were found to exhibit euchromatic features. In contrast, the subtelomeric regions and telomeric sequences present at interstitial chromosomal loci were heterochromatic. Six different epigenetic features were analyzed in this study, three that were heterochromatic (H3K9Me2, H3K27Me and DNA methylation) and three that were euchromatic (H3K4Me2, H3K9Ac and H4K16Ac). The telomeres were found to have lower levels of heterochromatic markers and higher levels of euchromatic markers relative to ITSs. In contrast, the subtelomeric regions and ITSs were enriched with heterochromatic markers and had low levels of euchromatic markers [13].

Although some concerns have been raised about this experimental procedure by Majerová and colleagues [21], these concerns are unjustified [22]. The results obtained using the Tru9I approach have been confirmed with the second technique mentioned above, which does not use frequently cutting restriction enzymes. This second approach is based on the analysis of the ChIP-seq data available in the publicly accessible databases [23]. It relies on the comparison of the number of perfect telomeric reads (at least four) with the number of centromeric reads. Four perfect tandem telomeric repeats are present in $98 \%$ of the cases at telomeres. This analysis revealed that Arabidopsis telomeres have lower levels of heterochromatic marks than centromeres (H3-K9Me2 and H3 $\mathrm{K} 27 \mathrm{Me}$ ), higher levels of some euchromatic marks (H3 $\mathrm{K} 4 \mathrm{Me} 2$ and H3K9Ac) and similar or lower levels of other euchromatic marks (H3K4Me3, H3K36Me2, H3K $36 \mathrm{Me} 3$ and H3K18Ac). Interestingly, the ChIP-seq experiments also revealed that Arabidopsis telomeres exhibit high levels of H3K27Me3, a repressive mark that associates with many euchromatic genes. This chromatin organization is found in 23\% of Arabidopsis genes, many of which are repressed or lowly expressed.

In general, the use of frequently cutting restriction enzymes could enable the study of other genomic repetitive sequences, if their composition varies at different genomic loci. In addition, the use of frequently cutting restriction enzymes or of ChIP-seq data could enable the study of telomeric chromatin structure in other model systems where ITSs are composed of degenerate telomeric repeats interspersed with short arrays of perfect telomeric repeats.

\section{ACKNOWLEDGEMENTS}

This work was supported by grant BIO2011-24794 from the Spanish Ministry of Economy and Innovation.

\section{REFERENCES}

[1] Blackburn, E. (2005) Telomeres and telomerase: Their mechanisms of action and the effects of altering their functions. FEBS Letters, 579, 859-862.

doi:10.1016/j.febslet.2004.11.036

[2] Cech, T. (2004) Beginning to understand the end of the chromosome. Cell, 43, 405-413.

[3] Greider, C. (2006) Telomerase RNA levels limit the telomere length equilibrium. Cold Spring Harbour Symposium on Quantitative Biology, 71, 225-229. doi:10.1101/sqb.2006.71.063

[4] Blasco, M. (2007) The epigenetic regulation of mammalian telomeres. Nature Reviews of Genetics, 8, 299-309. doi:10.1038/nrg2047

[5] Lundblad, V. and Blackburn, E. (1993) An alternative pathway for yeast telomere maintenance rescues est1 senescence. Cell, 73, 347-360. doi:10.1016/0092-8674(93)90234-H

[6] Riha, K., McKnight, T., Griffing, L. and Shippen, D. (2001) Living with genome instability: Plant responses to 
telomere dysfunction. Science, 291, 1797-1780. doi:10.1126/science. 1057110

[7] Shakirov, E., Surovtseva, Y., Osbun, N. and Shippen, D. (2005) The Arabidopsis Pot1 and Pot2 proteins function in telomere length homeostasis and chromosome end protection. Molecular and Cellular Biology, 25, 7725-7753. doi:10.1128/MCB.25.17.7725-7733.2005

[8] Vespa, L., Couvillion, M., Spangler, E. and Shippen, D. (2005) ATM and ATR make distinct contributions to chromosome end protection and the maintenance of telomeric DNA in Arabidopsis. Genes and Development, 19, 2111-2115. doi:10.1101/gad.1333805

[9] Aubert, G., Hills, M. and Lansdorp, P. (2012) Telomere length measurement-caveats and a critical assessment of the available technologies and tools. Mutation Research, 730, 59-67. doi:10.1016/j.mrfmmm.2011.04.003

[10] Gámez-Arjona, F., López-López, C., Vaquero-Sedas, M. and Vega-Palas, M. (2010) On the organization of the nucleosomes associated with telomeric sequences. Biochimica et Biophysica Acta, 1803, 1058-1061. doi:10.1016/j.bbamcr.2010.03.021

[11] Faravelli, M., Azzalin, C., Bertoni, L., Chernova, O., Attolini, C., Mondello, C. and Giulotto E. (2002) Molecular organization of internal telomeric sequences in Chinese hamster chromosomes. Gene, 283, 11-16. doi:10.1016/S0378-1119(01)00877-0

[12] Vaquero-Sedas, M. and Vega-Palas, M. (2011) On the chromatin structure of eukaryotic telomeres. Epigenetics, 6, 1055-1558. doi:10.4161/epi.6.9.16845

[13] Vaquero-Sedas, M., Gámez-Arjona, F. and Vega-Palas, M. (2011) Arabidopsis thaliana telomeres exhibit euchromatic features. Nucleic Acids Research, 39, 20072017. doi:10.1093/nar/gkq1119

[14] Richards, E. and Ausubel, F. (1988) Isolation of a higher eukaryotic telomere from Arabidopsis thaliana. Cell, 53, 127-136. doi:10.1016/0092-8674(88)90494-1
[15] Richards, E., Goodman, H. and Ausubel, F. (1991) The centromere region of Arabidopsis thaliana chromosome 1 contains telomere-similar sequences. Nucleic Acids Research, 19, 3351-3357. doi:10.1093/nar/19.12.3351

[16] Richards, E., Chao, S., Vongs, A. and Yang, J. (1992) Characterization of Arabidopsis thaliana telomeres isolated in yeast. Nucleic Acids Research, 20, 4039-4046. doi:10.1093/nar/20.15.4039

[17] Regad, F., Lebas, M. and Lescure, B. (1994) ITSs within the Arabidopsis thaliana genome. Journal of Molecular Biology, 239, 163-169. doi:10.1006/jmbi.1994.1360

[18] Uchida, W., Matsunaga, S., Sugiyama, R. and Kawano, S. (2002) Interstitial telomere-like repeats in the Arabidopsis thaliana genome. Genes Genetic Systems, 77, 63-67. doi:10.1266/ggs.77.63

[19] Shakirov, E. and Shippen, D. (2004) Length regulation and dynamics of individual telomere tracts in wild-type Arabidopsis. Plant Cell, 16, 1959-1967. doi:10.1105/tpc.104.023093

[20] Vannier, J., Depeiges, A., White, C. and Gallego, M. (2009) ERCC1/XPF protects short telomeres from homologous recombination in Arabidopsis thaliana. PLoS Genetics, 5, e1000380.

[21] Majerová, E., Fojtová, M., Mandáková, T. and Fajkus, J. (2011) Methylation of plant telomeric DNA: What do the results say? Plant Molecular Biology, 77, 533-536. doi:10.1007/s11103-011-9834-5

[22] Vaquero-Sedas, M. and Vega-Palas, M. (2012) The restriction endonuclease Tru9I is a useful tool for the analysis of telomeric chromatin structure in Arabidopsis thaliana. American Journal of Molecular Biology, 2, 242244. doi:10.4236/ajmb.2012.23025

[23] Vaquero-Sedas, M., Luo, C. and Vega-Palas, M. (2012) Analysis of the epigenetic status of telomeres by using ChIP-seq data. Nucleic Acids Research, 40, e163. doi:10.1093/nar/gks730 